\title{
Unipolar Codes for Spectral-Amplitude-Coding Optical CDMA Systems Based on Projective Geometries
}

\author{
Ivan B. Djordjevic and Bane Vasic, Senior Member, IEEE
}

\begin{abstract}
A novel class of unipolar optical orthogonal codes (OOCs), with ideal in-phase cross correlation for spectral-amplitude coding optical code-division multiple-access systems based on projective geometry (PG) is proposed. Large flexibility in cross-correlation control makes the proposed OOC family an excellent candidate for applications that require a large number of users. The construction permits arbitrary large $\mathrm{OOC}$ families. Novel electronically controllable PG-OOC encoder-decoder architectures based on arrayed waveguide grating and semiconductor optical amplifier gates are proposed as well.
\end{abstract}

Index Terms-Optical code-division multiple-access (CDMA), optical orthogonal codes (OOCs), projective geometries (PGs), spectral-amplitude coding.

\section{INTRODUCTION}

$\mathbf{T}$ HE MAIN factor of performance degradation in optical code-division multiple-access (CDMA) systems is the multiuser interference (MUI) [2]-[6]. In spectral-amplitude-coding (SAC) systems [2]-[6], MUI is solely a function of the in-phase cross correlations among the address sequences (also known as signature sequences or spreading sequence or simply code sequences). If the in-phase cross correlation among the address sequences is fixed, then the balanced detection receiver proposed in [3] is able to suppress MUI completely. Hadamard code $m$-sequence modified quadratic-congruence (MQC) code and modified frequency-hopping (MFH) codes have been proposed for such applications [5], [6].

The idea of using projective geometries (PGs) to construct optical orthogonal codes (OOCs) is not new. It was initially proposed in [1], however, with neither specific construction nor a specific application. In [4], the PGs were proposed for SAC optical CDMA systems, but no construction was given, and finally in [6], two PG constructions [8] were proposed with not necessarily ideal in-phase cross correlation (the example $q=2$, $m=3$, given in [6] has the in-phase cross correlation equal to three.) The first construction given in [6] requires solving a system of $\left(q^{m+1}-1\right) /(q-1)$ equations in Gallois field GF $(q)$ ( $q$-a prime power, $m>1$ ). The second construction in [6] gives only one code sequence, while the other sequences are cyclic shifts of the first one.

Manuscript received October 27, 2002; revised May 22, 2003

I. B. Djordjevic is with the Department of Electrical and Electronic Engineering, University of Bristol, Bristol BS8 1TR, U.K. (e-mail: Ivan.Djordjevic@bristol.ac.uk).

B. Vasic is with the Department of Electrical and Computer Engineering, University of Arizona, Tucson, AZ 85721 USA.

Digital Object Identifier 10.1109/LPT.2003.816672
The goal of this letter is to address: 1) construction of OOC and 2) encoder-decoder architecture. We propose a novel class of OOCs with ideal in-phase cross correlation based on PG. The geometry is defined as a collection of lines connecting the points from a finite set, satisfying a given set of axioms. Such a geometry has the following fundamental properties that can be exploited to construct fixed in-phase cross-correlation OOC: 1) every line consists of the same number of points; 2) any two points are connected by only one line; 3) two lines intersect at one and only one point; and 4) each point is an intersection of a fixed number of lines. From these properties it follows that the total number of points in PG $v$ determines codeword length, and the number of points in a line $k$ determines codeword weight, while the lines themselves determine the position of binary ones within a codeword. Since two lines intersect at exactly one point, the sequences have ideal in-phase cross correlation (equal to one).

Encoder-decoder schemes for SAC systems proposed so far were based on fiber Bragg grating (FBG) technology [3]. In order to change the address sequence in such a technology, piezoelectric or thermal tunability is required. Reconfigurability is limited by the tuning range of FBG. For narrower spectral slices, longer FGBs are required, which impose a limitation on the maximum supported bit rate, and giving rise to packaging issues. An encoding-decoding structure based on arrayed waveguide grating (AWG) has been proposed recently [7] in connection with time-wavelength hopping scheme. The expensive tunable delay lines are required for reconfigurability purposes.

We propose an AWG-based encoder structure that includes a set of semiconductor optical amplifier (SOA) gates, which enable an electronic switching between different address sequences with switching speed (in the order of $1 \mathrm{~ns}$ ) much higher than the temperature-driven switching mechanism in the FBGbased scheme (which is in the millisecond range). It is also faster, simpler, and more reliable than piezoelectric switching which requires microprocessor control and strict tolerance of the mechanical components. Another important feature of the proposed scheme is that the encoder-decoder can be readily integrated on silicon, and that it solves the power budget problem inherent with FBG scheme.

\section{OOC BASED ON PGs}

The code with fixed in-phase cross correlation between any two codewords (address sequences) is denoted as $(\nu, \kappa, \lambda)$, where $\nu$ and $\kappa$ are codeword length and weights, respectively, 
and $\lambda$ is the in-phase cross correlation. Since $\lambda=1$ is the minimum value that can be achieved, it is called here the ideal in-phase cross-correlation constraint, similarly as in [5] and [6]. PG designs allow both OOC families having ideal and nonideal in-phase cross correlation to be constructed.

The finite PGs PG $\left(m, p^{s}\right)$ are constructed using $(m+1)$-tuples $\mathbf{x}=\left(x_{0}, x_{1}, \ldots, x_{m}\right)$ of elements $x_{i}$ from $\operatorname{GF}\left(p^{s}\right)(p$-a prime, $s$-positive integer), not all simultaneously equal to zero, called points. Two $(m+1)$-tuples, $\mathbf{x}=\left(x_{0}, x_{1}, \ldots, x_{m}\right)$ and $\mathbf{y}=\left(y_{0}, y_{1}, \ldots, y_{m}\right)$, represent the same point if $\mathbf{y}=\lambda \mathbf{x}$, where $\lambda \in \operatorname{GF}\left(p^{s}\right)$ is a nonzero element. So each point can be represented on $p^{s}-1$ ways, referred to as the equivalence class. It is obvious, then, that the number of points in $\operatorname{PG}\left(m, p^{s}\right)$ is $v=\left[p^{(m+1) s}-1\right] /\left(p^{s}-1\right)$.

The equivalence classes (points) can be represented by $\left[\alpha^{i}\right]=\left\{\alpha^{i}, \beta \alpha^{i}, \beta^{2} \alpha^{i}, \ldots, \beta^{p^{s}-2} \alpha^{i}\right\},(0 \leq i \leq v)$, where $\beta=\alpha^{v}$. Let $\left[\alpha^{i}\right]$ and $\left[\alpha^{j}\right]$ be two distinct points in $\mathrm{PG}\left(m, p^{s}\right)$; then the line passing through them consists of points of the form $\left[\lambda_{1} \alpha^{i}+\lambda_{2} \alpha^{j}\right]$, where $\lambda_{1}, \lambda_{2} \in \operatorname{GF}\left(p^{s}\right)$. Since $\left[\lambda_{1} \alpha^{i}+\lambda_{2} \alpha^{j}\right]$ and $\left[\beta^{k} \lambda_{1} \alpha^{i}+\beta^{k} \lambda_{2} \alpha^{j}\right]$ are the same point, each line in $\mathrm{PG}\left(m, p^{s}\right)$ consists of $k=\left(p^{m s}-1\right) /\left(p^{s}-1\right)$ points. The number of lines intersecting at a given point is $\left(p^{m s}-1\right) /\left(p^{s}-1\right)$, and the number of lines in $\mathrm{PG}$ is $b=\left(p^{s(m+1)}-1\right)\left(p^{s m}-1\right) /\left[\left(p^{2 s}-1\right)\left(p^{s}-1\right)\right]$.

We now form the incidence matrix $A=\left(a_{i j}\right)_{b x \nu}$ whose rows correspond to the lines and columns to the points, with columns being arranged in the order $\left[\alpha^{0}\right],\left[\alpha^{1}\right], \ldots,\left[\alpha^{v}\right] . a_{i j}=1$ if $j$ th point belongs to $i$ th line, and zero otherwise. Each row has weight $p^{s}+1$ and each column $\left(p^{m s}-1\right) /\left(p^{s}-1\right)$. Any two columns have exactly one " 1 " in common, and any two rows at most one " 1 " in common. Therefore, the columns of the incident matrix represent different codewords of an OOC having the ideal in-phase cross correlation.

\section{ENCODER-DECODER STRUCTURE}

Recently, an encoder-decoder structure for the SAC system was proposed by Wei et al. [3]. It requires two sets of FBGs with opposite order to compensate the delay of different portions of the broad-band spectrum. This structure can be simplified by using one set of FBGs in the transmitter and the same set of FBGs in the receiver put in opposite order (to compensate delay). In order to change the codeword sequence, each particular FBG must be tuned piezoelectrically or thermally.

We propose the structure based on AWG and SOA gates, shown in Fig. 1(a). Incoming broad-band source pulse is spectrally sliced using AWG. Those wavelengths (spectral slices) corresponding to the position of ones within a codeword are amplified by SOA gates, while the other wavelengths are blocked. Provided that different wavelengths experience the same delays, the amplified wavelengths are fed back to the input side of AWG at specific ports so that they all recombine on the same output port (encoder-decoder output). The codeword signature is easy to change by turning on-off the SOA gates according to the position of ones within a codeword. Since SOA gates also serve as amplifiers, a much better power budget can be achieved with the proposed structure. An integration on silicon technology is possible, too. Any other optical switch can be used instead of SOA gate. Alternatively, SOA gates may be replaced by variable optical attenuators, but the power budget advantage will be lost.

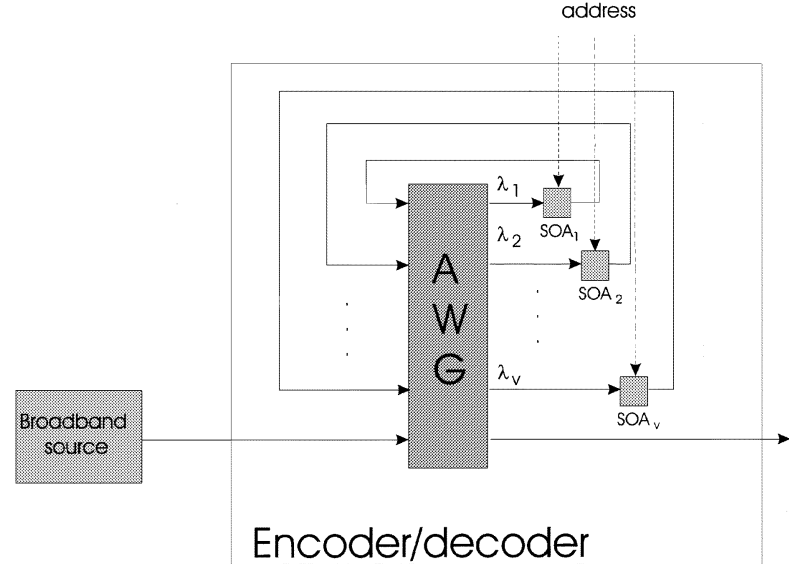

(a)

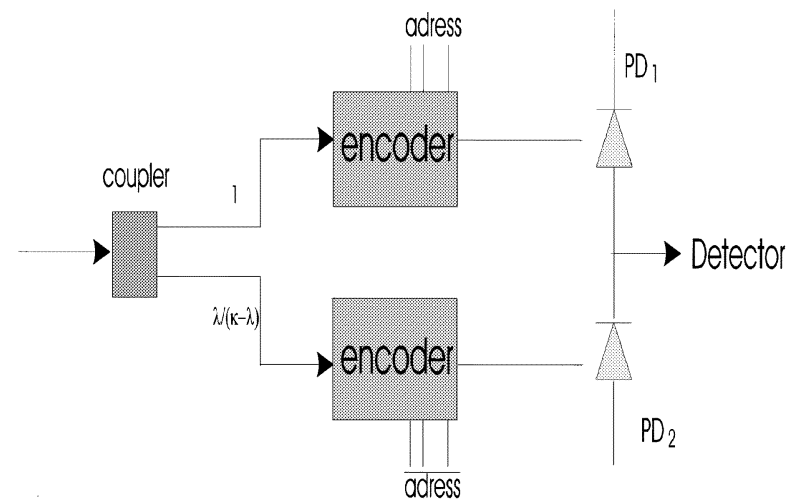

(b)

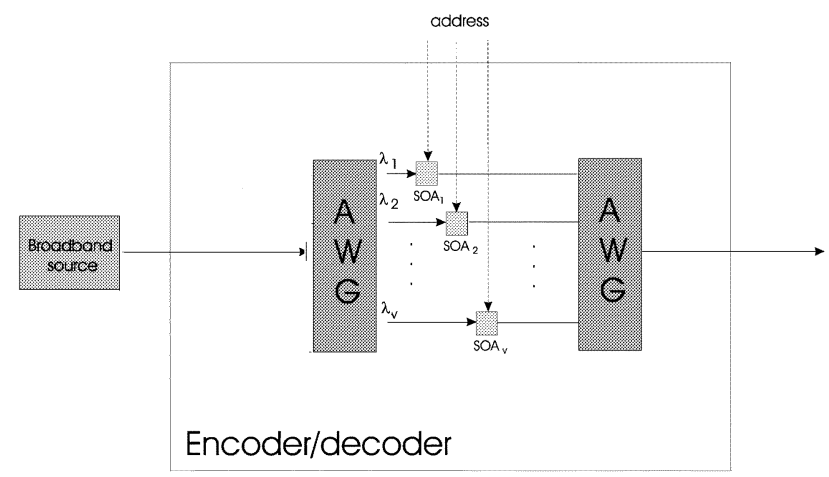

(c)

Fig. 1. (a) Encoder structure for a single photodiode SAC receiver (b) Balanced receiver. (c) Encoder structure insensitive to bit rate.

For balanced reception (capable of suppressing the MUI), we propose the receiver structure shown in Fig. 1(b). The received signal is divided into two parts using $1: \lambda /(k-\lambda)$ splitter whose outputs are then used as inputs of two encoders with complementary functions, determined by the address sequence. The MUI at the first photodiode $\left(\mathrm{PD}_{1}\right)(N-1) \lambda$ (where $N$ is the number of active users), and that of the second photodiode $\left(\mathrm{PD}_{2}\right)[\lambda /(k-\lambda)][(k-\lambda)(N-1)]=(N-1) \lambda$ are the same, and can be, therefore, successfully removed by balanced detection.

Similarly, as his counterpart FBG-based encoders, the encoder proposed in Fig. 1(a) has a disadvantage that only a limited bit rate can be supported. Namely, the encoded broad-band pulse must leave the encoder before the next pulse 
comes from broad-band source. The simplest way is to made modification to the encoder, as proposed in Fig. 1(c). This structure is insensitive to the bit rate of the incoming data, and can also be fabricated in silicon technology. It also allows for an easier minimization of the delay among different spectrally sliced components. Another approach is to put SOA gates in the waveguide-grating region, but such an approach requires detailed study. Any other wavelength-division multiplexing demultiplexer-multiplexer pair can be used instead of AWG pairs. From a coding theory point of view, the encoder structure from Fig. 1(a) can support higher bit rates if the input sequence is coded by a $(d, k)$ constrained code similar to ones developed for optical recording systems [9]. Namely, we are able to insert zeros between ones on a controlled manner to avoid successive ones, since broad-band source emits a pulse for every mark-state bit (and nothing is emitted for space-state bits).

\section{Performance Analysis}

All classes of OOCs based on BIBD can be designed to have comparable performance. Two such classes of OOC, namely MFH [3], [6], [7], and MQC [4], [6], [7], have been proposed recently. However, our code families have much larger flexibility in choosing the number of users. This is a consequence of the fact that in our constructions three parameters $(m, p$, and $s)$ can be varied, as opposed to just one free parameter ( $p$ or $Q)$, in MQC or MFH.

The performance of the PG OOC family and the previously reported MFH [5], [6], the MQC [3], [5], [6], and the Hadamard code [2] in the presence of the phase-induced intensity noise, the photodiode shot noise, and the thermal noise are illustrated in Fig. 2(a). The system parameters are: $\Delta \nu=3.75 \mathrm{THz}$, $B=311 \mathrm{MHz}$, bit rate $622 \mathrm{Mb} / \mathrm{s}$, central wavelength $1550 \mathrm{~nm}$, $T_{r}=300 \mathrm{~K}, R_{L}=1030 \Omega$, photodiode quantum efficiency 0.6 . PG $(2,16)$ has the same performance as the previously reported MFH $(Q=16)$ but supports a larger number of users. (The same notation as in [2] is applied). The bit-error rate can be calculated using the Gaussian approximation [2]-[6]. PG designs with performance close to MQC, giving nonideal cross correlation but supporting even larger numbers of users, are also shown in Fig. 2(a). Fig. 2(b) shows that the performance of the MFH and MQC OOC families for $2.5-\mathrm{Gb} / \mathrm{s}$ channels is similar to the proposed OOC (the PG curve overlaps with the MFH curve). However, the PG OOC supports a larger number of users (MFH cardinality is 256, while PG cardinality is 273). For BER of $10^{-9}$, the system supports $50622-\mathrm{Mb} / \mathrm{s}$ [Fig. 2(a)] and 17 2.5-Gb/s [Fig. 2(b)] channels resulting in the capacity of 31 and $42.5 \mathrm{~Gb} / \mathrm{s}$, respectively. A system with a forward error correction (FEC) based on RS(255 239) supports at least $502.5-\mathrm{Gb} / \mathrm{s}$ error-free channels leading to the capacity of at least $125 \mathrm{~Gb} / \mathrm{s}$. Much larger capacities are achievable with more advanced FEC techniques such as low-density parity-check codes [10].

\section{CONCLUSION}

A novel design of OOCs for spectral-amplitude coding with ideal in-phase cross correlation based on PG has been proposed. It offers larger flexibility in choosing the number of users then previously reported OOC, and has a simple construction al-
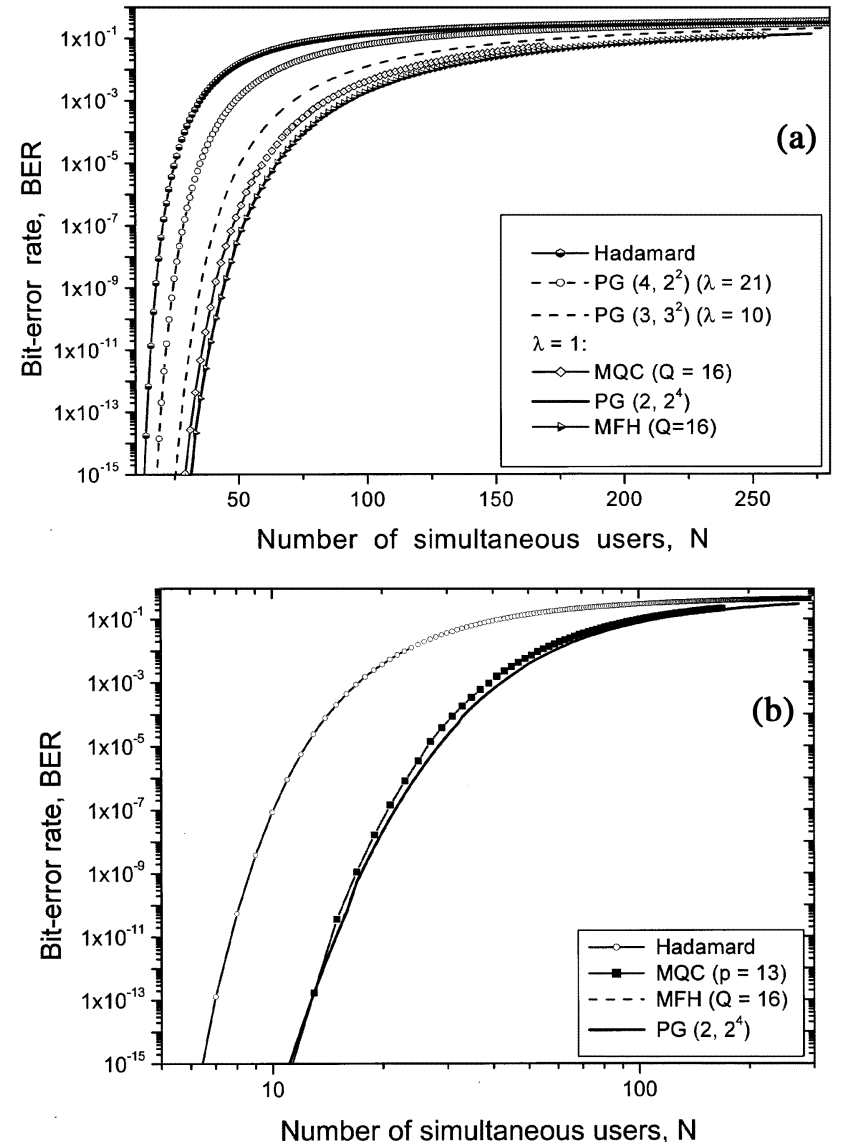

Fig. 2. Bit-error rate versus number of active users for different $\mathrm{OOC}$ families and two different channel bit rates. (a) $622 \mathrm{Mb} / \mathrm{s}$. (b) $2.5 \mathrm{~Gb} / \mathrm{s}$.

gorithm. The codes designed using our method have performance comparable with previously reported MFH, but support a larger number of users. Novel encoder-decoder with the possibility of selection of the desired codeword electronically is also proposed.

\section{REFERENCES}

[1] F. R. K. Chung et al., "Optical orthogonal codes: Design, analysis, and applications," IEEE Trans. Inform. Theory, vol. 35, pp. 595-604, May 1989.

[2] E. D. J. Smith et al., "Performance enhancement of spectral-amplitudecoding optical CDMA using pulse-position modulation," IEEE Trans. Commun., vol. 46, pp. 1176-1185, Sept. 1998.

[3] Z. Wei et al., "Modified quadratic congruence codes for fiber Bragg-grating-based spectral-amplitude-coding optical CDMA systems," J. Lightwave Technol., vol. 19, pp. 1274-1281, Sept. 2001.

[4] X. Zhou et al., "Code for spectral amplitude coding optical CDMA systems," Electron. Lett., vol. 36, pp. 728-729, 2000.

[5] Z. Wei and H. Ghafouri-Shiraz, "Unipolar codes with ideal in-phase cross correlation for spectral amplitude-coding optical CDMA systems," IEEE Trans. Commun., vol. 50, pp. 1209-1212, Aug. 2002.

[6] Z. Wei et al., "Codes for spectral-amplitude-coding optical CDMA systems," J. Lightwave Technol., vol. 20, pp. 1284-1291, Aug. 2002.

[7] S. Yengnarayanan, A. S. Bhushan, and B. Jalali, "Fast wavelength-hopping time spreading encoding-decoding for optical CDMA," IEEE Photon. Technol. Lett., vol. 12, pp. 573-575, May 2000.

[8] I. Andersen, Combinatorial Designs: Construction Methods. New York: Wiley, 1990.

[9] K. A. S. Immink, "Runlength-limited sequences," Proc. IEEE, vol. 78, pp. 1745-1759, Nov. 1990 .

[10] B. Vasic, I. B. Djordjevic, and R. Kostuk, "Low-density parity check codes and iterative decoding for long Haul optical communication systems," J. Lightwave Technol., vol. 21, pp. 438-446, Feb. 2003. 